УДК 532.5.013.4

\title{
A Priori Estimates of the Conjugate Problem Describing an Axisymmetric Thermocapillary Motion for Small Marangoni Number
}

\author{
Victor K. Andreev* \\ Evgeniy P. Magdenko ${ }^{\dagger}$ \\ Institute of Computational Modelling SB RAS \\ Akademgorodok, 50/44, Krasnoyarsk, 660036 \\ Institute of Mathematics and Fundamental Informatics \\ Siberian Federal University \\ Svobodny, 79, Krasnoyarsk, 660041 \\ Russia
}

Received 28.02.2019, received in revised form 10.03.2019, accepted 12.06.2019

This paper is devoted to the study of equations solution describing the axisymmetric motion of a viscous heat-conducting liquid. The motion is interpreted as a two-layer flow of viscous heat-conducting liquids in a cylinder with a solid wall and a common movable non-deformable interface. From a mathematical point of view, the arising initial-boundary value problem is nonlinear and inverse. Under certain assumptions concerning to apply the problem is replaced by a linear one. As a result, the unimprovable uniform priori estimates for solutions of the problems posed are obtained.

Keywords: a priori estimates, the conjugate inverse problem, interface, Marangoni number.

DOI: 10.17516/1997-1397-2019-12-4-483-495.

\section{Introduction}

It is well known that a motion can arise in a non-uniformly heated liquid. In some applications of liquid flows, a joint motion of two or more fluids with surfaces takes place. If the liquids are not soluble in each other, they form a more or less visual interfaces. The petroleum-water system is a typical example of this situation. At the present time modelling of multiphase flows taking into account different physical and chemical factors is needed for designing of cooling systems and power plants, in biomedicine, for studying the growth of crystals and films, in aerospace industry [1-4].

The stationary solution of the Navier-Stokes equations describing the $2 \mathrm{D}$ motion of a pure viscid incompressible heat-conducting fluids in the absence of mass forces was found for the first time by [5]. It describes the liquid impingement from infinity on the plane under the no slip condition on it. In the paper [6], this solution for the flow between two plates or for the flow in a cylindrical tube was applied.

The monograph [7] presents the results of specific non-stationary motions studies of a binary mixture with allowance for the effect of thermal diffusion arising in sufficiently long plane and

\footnotetext{
*andr@icm.krasn.ru

† magdenko_evgeniy@icm.krasn.ru

(c) Siberian Federal University. All rights reserved
} 
cylindrical layers. The properties of invariant solutions of thermal diffusion equations are considered, when the surface tension on the interface of two mixtures depends linearly on temperature and concentration. A generalization of the Ostroumov-Birich solutions to the motion of mixtures in a cylindrical tube is given.

\section{The problem statement}

We will consider at the following linear conjugate inverse initial-boundary problem

$$
\begin{gathered}
v_{1 t}=\nu_{1}\left(v_{1 r r}+\frac{1}{r} v_{1 r}\right)+f_{1}(t), \quad 0<r<R_{1}, \\
v_{2 t}=\nu_{2}\left(v_{2 r r}+\frac{1}{r} v_{2 r}\right)+f_{2}(t), \quad R_{1}<r<R_{2}, \\
v_{1}\left(R_{1}, t\right)=v_{2}\left(R_{1}, t\right), \quad \int_{0}^{R_{1}} r v_{1}(r, t) d r+\int_{R_{1}}^{R_{2}} r v_{2}(r, t) d r=0, \\
\mu_{1} v_{1 r}\left(R_{1}, t\right)-\mu_{2} v_{2 r}\left(R_{1}, t\right)=-2 æ a_{1}\left(R_{1}, t\right), \\
\left|v_{1}(0, t)\right|<\infty, \quad v_{2}\left(R_{2}, t\right)=0, \\
v_{1}(r, 0)=0, \quad v_{2}(r, 0)=0, \\
\rho_{1} f_{1}(t)=\rho_{2} f_{2}(t)-\frac{2 æ a_{1}\left(R_{1}, t\right)}{R_{1}}
\end{gathered}
$$

and the closed conjugate problem for functions $a_{j}(r, t)$ is described the following equations:

$$
\begin{gathered}
a_{j t}=\chi_{j}\left(a_{j r r}+\frac{1}{r} a_{j r}\right), \\
a_{j}(r, 0)=a_{j}^{0}(r), \quad\left|a_{1}(0, t)\right|<\infty, \\
a_{2}\left(R_{2}, t\right)=\alpha(t), \\
a_{1}\left(R_{1}, t\right)=a_{2}\left(R_{1}, t\right), \quad k_{1} a_{1 r}\left(R_{1}, t\right)=k_{2} a_{2 r}\left(R_{2}, t\right) .
\end{gathered}
$$

Here $\mu_{j}$ is dynamic viscosity coefficient (index $j=1,2$ is number of the liquid), $\rho_{j}$ is the density, $æ=-d \sigma / d \theta=$ const, $\sigma(\theta)$ is the surface tension coefficient, $\chi_{j}$ и $k_{j}$ are the coefficients of thermal diffusivity and conductivity, respectively. The functions $v_{1}(r, t), a_{1}(r, t)$ are limited at $r=0$ for all values of time. The system (1.1)-(1.11) describes the two-layer motion of viscous heat-conducting fluids in a cylinder with a solid side surface $r=R_{2}=$ const and the common interface $r=h(t), 0<h(t)<R_{2}$.

The problem (1.1)-(1.11) is obtained from general equations describing the axisymmetric motion of a viscous heat-conducting fluid in the absence of mass forces, if the Marangoni number $\mathrm{M}=æ a^{1} R_{1}^{3} / \mu_{1} \chi_{1} \rightarrow 0$. Here $a^{1}=\max _{t \in[0, T]}|\alpha(t)|$. The function $\alpha(t)$ is bounded in physical sense for all $t$ from the interval $[0, T]$, where $T$ is constant. The constant $a^{1} R_{2}^{2}$ is the characteristic temperature along a solid wall. In this case, the velocity, pressure, and temperature fields are described by the formulas

$$
u_{j}=u_{j}(r, t), \quad w_{j}=v_{j}(r, t) z, \quad p_{j}=p_{j}(r, z, t), \quad \theta_{j}=\theta_{j}(r, z, t),
$$

where $u_{j}(r, z, t)$ and $w_{j}(r, z, t)$ are the projection of the velocity vector on the axis $r$ and $z$ of the cylindrical coordinate system, $p_{j}(r, z, t)$ is the pressure that satisfies the relation

$$
\frac{1}{\rho_{j}} p_{j}=d_{j}(r, t)-\frac{f_{j}(t)}{2} z^{2}, \quad d_{j r}=\nu_{j}\left(u_{j r r}+\frac{1}{r} u_{j r}-\frac{u_{j}}{r^{2}}\right)-u_{j t}-u_{j} u_{j r} .
$$


The temperature field is sought in the form

$$
\theta_{j}(r, z, t)=a_{j}(r, t) z^{2}+b_{j}(r, t) .
$$

The temperature has extreme at the point $z=0$. It has a maximum at $a(r, t)<0$ and minimum at $a(r, t)>0$.

We note that the problem posed is inverse, since together with $v_{j}(r, t), a_{j}(r, t), b_{j}(r, t), h(t)$ the functions $f_{j}(t)$ should be found. The functions $u_{j}(r, t)$ are determined by the equalities

$$
u_{1}(r, t)=-\frac{1}{r} \int_{0}^{r} r v_{1}(r, t) d r, \quad u_{2}(r, t)=\frac{1}{r} \int_{r}^{R_{2}} r v_{2}(r, t) d r .
$$

With known $u_{j}(r, t), a_{j}(r, t)$, the problem for functions $b_{j}(r, t)$ is separated. The functions $d_{j}(r, t)$ are reconstructed by quadratures from (1.13). The interface is described by the formula

$$
h(t)=R_{1}\left[1+\mathrm{M} h_{1}(t)\right], \quad h_{1}(t)=-\frac{1}{R_{1}} \int_{0}^{t} r v_{1}\left(R_{1}, t\right) d t .
$$

Remark 1. The second equation in (1.3) and the final formula (1.7) allow us to find the pressure gradients along the axis $z, i$. e., the functions $f_{j}(t)$.

\section{Estimates of the function $a_{j}(r, t)$}

Since the function $a_{1}\left(R_{1}, t\right)$ is included in the statement of the problem for $v_{j}(r, t)$, it is necessary to begin with an estimate $a_{j}(r, t)$ satisfying the initial boundary-value problem (1.8)-(1.11). For smooth solutions, the following matching conditions should be satisfied:

$$
\begin{aligned}
a_{2}^{0}\left(R_{2}\right) & =\alpha(0), \quad a_{1}^{0}\left(R_{1}\right)=a_{2}^{0}\left(R_{1}\right), \\
k_{1} a_{1 r}^{0}\left(R_{1}\right) & =k_{2} a_{2 r}^{0}\left(R_{1}\right), \quad\left|a_{1}^{0}(0)\right|<\infty .
\end{aligned}
$$

Perform a replacement

$$
a_{2}(r, t)=\bar{a}_{2}(r, t)+\frac{\alpha(t)\left(r-R_{1}\right)^{2}}{\left(R_{2}-R_{1}\right)^{2}} .
$$

Then the boundary condition (1.10) for the function $\bar{a}_{2}(r, t)$ becomes homogeneous. It satisfies the inhomogeneous equation

$$
\bar{a}_{2 t}-\chi_{2}\left(\bar{a}_{2 r r}+\frac{1}{r} \bar{a}_{2 r}\right)=\frac{2 \chi_{2} \alpha(t)}{\left(R_{2}-R_{1}\right)^{2}}\left(2-\frac{R_{1}}{r}\right)-\frac{\alpha^{\prime}(t)\left(r-R_{1}\right)^{2}}{\left(R_{2}-R_{1}\right)^{2}} \equiv g_{2}(r, t),
$$

where the prime denotes differentiation with respect to $t$. Conditions (1.11) for the functions $\bar{a}_{2}(r, t), a_{1}(r, t)$ remain unchanged.

We multiply equation (1.8) $(j=1)$ by $\rho_{1} c_{p_{1}} r a_{1}(r, t)$ and multiply equation (2.4) by $\rho_{2} c_{p_{2}} r a_{2}(r, t)$, where $c_{p_{j}}$ are the specific heat of liquids at constant pressure. Then, integration the equation obtained over the intervals of definition and adding up, leads to the integral equality $\left(k_{j}=\rho_{j} c_{p_{j}} \chi_{j}\right)$

$$
\frac{d}{d t} A+k_{1} \int_{0}^{R_{1}} r a_{1 r}^{2} d r+k_{2} \int_{R_{1}}^{R_{2}} r \bar{a}_{2 r}^{2} d r=\int_{R_{1}}^{R_{2}} r \bar{a}_{2} g_{2}(r, t) d r
$$


with function

$$
A(t)=\frac{\rho_{1} c_{p_{1}}}{2} \int_{0}^{R_{1}} r a_{1}^{2}(r, t) d r+\frac{\rho_{2} c_{p_{2}}}{2} \int_{R_{1}}^{R_{2}} r \bar{a}_{2}^{2}(r, t) d r .
$$

The solutions of equations (1.8) are sought in the intervals: $0<r<R_{1}$ at $j=1$ and $R_{1}<r<R_{2}$ at $j=2$ at $\mathrm{M} \rightarrow 0$. We have the inequality [8]

$$
\int_{0}^{R_{1}} r a_{1}^{2} d r+\int_{R_{1}}^{R_{2}} r \bar{a}_{2}^{2} d r \leqslant M_{0}\left(k_{1} \int_{0}^{R_{1}} r a_{1 r}^{2} d r+k_{2} \int_{R_{1}}^{R_{2}} r \bar{a}_{2 r}^{2} d r\right)
$$

with positive constant $M_{0}$.

We obtain that using inequality (2.7), the left-hand side of equation (2.5) is greater than or equal to

$$
\frac{d}{d t} A+\frac{1}{M_{0}}\left(\int_{0}^{R_{1}} r a_{1}^{2} d r+\int_{R_{1}}^{R_{2}} r \bar{a}_{2}^{2} d r\right)
$$

For the right-hand side we have estimate from above

$$
\begin{aligned}
& \int_{R_{1}}^{R_{2}} r \bar{a}_{2} g_{2} d r \leqslant\left(\int_{R_{1}}^{R_{2}} r g_{2}^{2} d r\right)^{1 / 2}\left(\int_{R_{1}}^{R_{2}} r \bar{a}_{2}^{2} d r\right)^{1 / 2} \leqslant \\
& \leqslant \max _{j}\left(\frac{2}{\rho_{j} c_{p_{j}}}\right)^{1 / 2}\left(\int_{R_{1}}^{R_{2}} r g_{2}^{2} d r\right)^{1 / 2} \sqrt{A} \equiv G(t) \sqrt{A} .
\end{aligned}
$$

Further we obtain the inequality from inequalities (2.8) and (2.9)

$$
\frac{d}{d t} A+2 \eta A \leqslant G(t) \sqrt{A}, \quad \eta=\frac{1}{M_{0}} \min _{j}\left(\frac{1}{\rho_{j} c_{p_{j}}}\right)=\frac{1}{M_{0}} \min _{j}\left(\frac{\chi_{j}}{k_{j}}\right) .
$$

Then from (2.10) it follows that

$$
A(t) \leqslant\left(\sqrt{A_{0}}+\frac{1}{2} \int_{0}^{t} G(\tau) e^{\eta \tau} d \tau\right)^{2} e^{-2 \eta t}
$$

here $A_{0}$ is value of function $A(t)$ at $t=0$ :

$$
\begin{gathered}
A(0)=\frac{\rho_{1} c_{p_{1}}}{2} \int_{0}^{R_{1}} r\left(a_{1}^{0}\right)^{2}(r) d r+\frac{\rho_{2} c_{p_{2}}}{2} \int_{R_{1}}^{R_{2}} r\left(\bar{a}_{2}^{0}\right)^{2}(r) d r, \\
\bar{a}_{2}^{0}(r)=a_{2}^{0}(r)-\frac{\alpha_{0}\left(r-R_{1}\right)^{2}}{\left(R_{2}-R_{1}\right)^{2}}, \quad \alpha_{0}=\alpha(0) .
\end{gathered}
$$

Therefore, from (2.6) we have

$$
\int_{0}^{R_{1}} r a_{1}^{2}(r) d r \leqslant \frac{2}{\rho_{1} c_{p_{1}}} A(t), \quad \int_{R_{1}}^{R_{2}} r \bar{a}_{2}^{2}(r) d r \leqslant \frac{2}{\rho_{2} c_{p_{2}}} A(t),
$$

where $A(t)$ satisfies the estimate (2.11). Let us prove the limitations of the integrals

$$
\int_{0}^{R_{1}} r a_{1 r}^{2} d r, \quad \int_{R_{1}}^{R_{2}} r \bar{a}_{2 r}^{2} d r
$$

To do this, we raise equations (1.8) $(j=1),(2.4)$ to the second degree then multiply them by $\rho_{1} c_{p_{1}} r, \rho_{2} c_{p_{2}} r$ respectively, integrate over their domains $r$ and $t$, we sum the results. We obtain 
another integral identity

$$
\begin{gathered}
\rho_{1} c_{p_{1}} \int_{0}^{t} \int_{0}^{R_{1}} r\left[a_{1 t}^{2}+\chi_{1}^{2}\left(a_{1 r r}+\frac{1}{r} a_{1 r}\right)^{2}\right] d r d t+ \\
+\rho_{2} c_{p_{2}} \int_{0}^{t} \int_{R_{1}}^{R_{2}}\left[\bar{a}_{2 t}^{2}+\chi_{2}^{2}\left(\bar{a}_{2 r r}+\frac{1}{r} \bar{a}_{2 r}\right)^{2}\right] d r d t+ \\
+k_{1} \int_{0}^{R_{1}} r a_{1 r}^{2} d r+k_{2} \int_{R_{1}}^{R_{2}} r \bar{a}_{2 r}^{2} d r=k_{1} \int_{0}^{R_{1}} r\left(a_{1 r}^{0}\right)^{2} d r+k_{2} \int_{R_{1}}^{R_{2}} r\left(\bar{a}_{2 r}^{0}\right)^{2} d r+ \\
+\rho_{1} c_{p_{1}} \int_{0}^{t} \int_{R_{1}}^{R_{2}} r g_{1}(r, t) d r d t+\rho_{2} c_{p_{2}} \int_{0}^{t} \int_{R_{1}}^{R_{2}} r g_{2}(r, t) d r d t \equiv A_{1}(t) .
\end{gathered}
$$

From (2.16) the limitation of the integrals (2.15) follows $\forall t \in[0, T]$.

Further, taking into account (2.14), (2.16), we have the inequality

$$
\begin{gathered}
\bar{a}_{2}^{2}(r, t)=\left|\int_{r}^{R_{2}}\left(\bar{a}_{2}^{2}\right)_{r} d r\right| \leqslant 2 \int_{R_{1}}^{R_{2}} \frac{1}{r} \sqrt{r}\left|\bar{a}_{2}\right| \sqrt{r}\left|\bar{a}_{2 r}\right| d r \leqslant \\
\leqslant \frac{2}{R_{1}}\left(\int_{R_{1}}^{R_{2}} r \bar{a}_{2}^{2} d r\right)^{1 / 2}\left(\int_{R_{1}}^{R_{2}} r a_{2 r}^{2} d r\right)^{1 / 2} \leqslant \frac{4}{R_{1}}\left(\frac{1}{k_{2} \rho_{2} c_{\rho_{2}}} A(t) A_{1}(t)\right)^{1 / 2} .
\end{gathered}
$$

Hence we obtain the estimate

$$
\left|\bar{a}_{2}(r, t)\right| \leqslant 2\left(\frac{1}{R_{1}^{2} k_{2} \rho_{2} c_{\rho_{2}}} A(t) A_{1}(t)\right)^{1 / 4}
$$

uniformly for all $r \in\left[R_{1}, R_{2}\right], t \in[0, T]$.

From the replacement (2.3) we find the estimate

$$
\left|a_{2}(r, t)\right| \leqslant|\alpha(t)|+2\left(\frac{1}{R_{1}^{2} k_{2} \rho_{2} c_{\rho_{2}}} A(t) A_{1}(t)\right)^{1 / 4} .
$$

Remark 2. If we differentiate with respect to time equations (1.8) $(j=1),(2.4)$ and the boundary conditions, then in the same way we obtain the estimate

$$
\left|a_{2 t}(r, t)\right| \leqslant\left|\alpha^{\prime}(t)\right|+2\left(\frac{1}{R_{1}^{2} k_{2} \rho_{2} c_{\rho_{2}}} A_{2}(t) A_{3}(t)\right)^{1 / 4},
$$

where the function $A_{2}(t)$ differs from $A(t)$ in that the function $a_{1}(r, t), \bar{a}_{2}(r, t)$ should be replaced by $a_{1 t}(r, t), \bar{a}_{2 t}(r, t)$ in formula (2.6). Similar the function $A_{3}(t)$ differences from $A_{1}(t)$.

Also,

$$
\left|a_{2 t t}(r, t)\right| \leqslant\left|\alpha^{\prime \prime}(t)\right|+2\left(\frac{1}{R_{1}^{2} k_{2} \rho_{2} c_{\rho_{2}}} A_{4}(t) A_{5}(t)\right)^{1 / 4} .
$$

Here the function $A_{4}(t)$ differs from $A(t)$ in that, in (2.6) their second derivatives with respect to time from functions $a_{1}(r, t), \bar{a}_{2}(r, t)$ are instead of this functions. The function $A_{5}(t)$ is quite similar to $A_{1}(t)$, however, $g_{2}(r, t)$ from (2.4) contains second derivative $\alpha^{\prime \prime}(t)$ instead of function $\alpha(t)$, and third derivative $\alpha^{\prime \prime \prime}(t)$ instead of function $\alpha^{\prime}(t)$. The estimates for (2.20) and (2.21) are used later in step 4 , where also the expressions for $A_{k}(t), k=2,3,4,5$ are given. Here we note that these functions are continuous for $t \in[0, T]$, if the function $\alpha^{(n)}(t), n=0,1,2,3$ are also continuous in the same interval. Moreover, $A_{2}(t), A_{4}(t)$ satisfy inequalities like (2.11) with their functions $G(t)$. 
However, such arguments are not suitable for estimate $\left|a_{1}(r, t)\right|$. From inequality (2.19) and first equality (1.11) we have the estimate

$$
\left|a_{1}\left(R_{1}, t\right)\right|=\left|a_{2}\left(R_{1}, t\right)\right| \leqslant|\alpha(t)|+2\left(\frac{1}{R_{1}^{2} k_{2} \rho_{2} c_{\rho_{2}}} A(t) A_{1}(t)\right)^{1 / 4} .
$$

For $a_{1}(r, t)$ we obtain the problem at $0<r<R_{1}$

$$
\begin{gathered}
a_{1 t}=\chi_{1}\left(a_{1 r r}+\frac{1}{r} a_{1 r}\right), \\
a_{1}\left(R_{1}, t\right)=a_{2}\left(R_{1}, t\right), \quad\left|a_{1}(0, t)\right|<\infty, \\
a_{1}(r, 0)=a_{1}^{0}(r) .
\end{gathered}
$$

The initial boundary value problem for equation $(2.23)-(2.25)$ at given $a_{1}\left(R_{1}, t\right)$ with estimate(2.22) has the solution [9]

$$
\begin{gathered}
a_{1}(r, t)=\frac{2 \chi_{1}}{R_{1}^{2}} \int_{0}^{t} a_{1}\left(R_{1}, \tau\right) \sum_{n=1}^{\infty} \frac{\xi_{n} J_{0}\left(\xi_{n} r / R_{1}\right)}{J_{1}\left(\xi_{n}\right)} \exp \left[-\frac{\chi_{1} \xi_{n}^{2}(t-\tau)}{R_{1}^{2}}\right] d \tau+ \\
+\frac{2}{R_{1}^{2}} \int_{0}^{R_{1}} \zeta a_{1}^{0}(\zeta) \sum_{n=1}^{\infty} \frac{J_{0}\left(\xi_{n} r / R_{1}\right) J_{0}\left(\xi_{n} \zeta / R_{1}\right)}{J_{1}^{2}\left(\xi_{n}\right)} \exp \left(-\frac{\chi_{1} \xi_{n}^{2} t}{R_{1}^{2}}\right) d \zeta,
\end{gathered}
$$

where $\xi_{n}$ are the roots of equation $J_{0}(\xi)=0$. From estimate (2.22) and from formula $(2.26)$ there is the limitation $\left|a_{1}(r, t)\right|$ for all $r \in\left[0, R_{1}\right]$ and $t \in[0, T]$. Indeed, the first term in $(2.26)$ is less than or equal to

$$
2\left[\max _{t \in[0, T]}|\alpha(t)|+\frac{1}{\left(R_{1}^{2} k_{2} \rho_{2} c_{\rho_{2}}\right)^{1 / 4}} \max _{t \in[0, T]}\left(A(t) A_{1}(t)\right)^{1 / 4}\right] .
$$

Under derivation of expression (2.27) we use inequality (2.22) and the relation [10]

$$
\sum_{n=1}^{\infty} \frac{J_{0}\left(\xi_{n} r / R_{1}\right)}{\xi_{n} J_{1}\left(\xi_{n}\right)}=\frac{1}{2}, \quad 0 \leqslant r<R_{1}
$$

Second summand in (2.26) does not exceed

$$
\max _{r \in\left[0, R_{1}\right]}\left|a_{1}^{0}(r)\right|
$$

We have proved

Lemma 2.1. The solution of the initial-boundary value problem (1.8)-(1.11) is limited for all $r \in\left[0, R_{1}\right](j=1)$ and $r \in\left[R_{1}, R_{2}\right](j=2)$, and $t \in[0, T]$. The estimates are the following

$$
\begin{aligned}
& \left|a_{1}(r, t)\right| \leqslant 2\left[\max _{t \in[0, T]}|\alpha(t)|+\frac{1}{\left(R_{1}^{2} k_{2} \rho_{2} c_{\rho_{2}}\right)^{1 / 4}} \max _{t \in[0, T]}\left(A(t) A_{1}(t)\right)^{1 / 4}\right]+\max _{r \in\left[0, R_{1}\right]}\left|a_{1}^{0}(r)\right|, \\
& \left|a_{2}(r, t)\right| \leqslant|\alpha(t)|+2\left(\frac{1}{R_{1}^{2} k_{2} \rho_{2} c_{\rho_{2}}} A(t) A_{1}(t)\right)^{1 / 4}
\end{aligned}
$$

with function $A(t), A_{1}(t)$ from (2.6) and (2.16) respectively. 


\section{Estimates of the function $v_{j}(r, t)$}

We turn to obtaining a priori estimates of the functions $v_{j}(r, t)$ that satisfy the equations (1.1), (1.2), boundary conditions (1.3)-(1.5) and initial data (1.6). In order to make boundary condition (1.4) homogeneous, we make the change of the function $v_{2}(r, t)$

$$
v_{2}(r, t)=\bar{v}_{2}(r, t)-\frac{2 æ a_{1}\left(R_{1}, t\right)}{\mu_{2}} P_{4}(r) .
$$

The polynomial of the fourth order $P_{4}(r)$ satisfies the following conditions: 1) $P_{4}\left(R_{1}\right)=0$, $\left.P_{4}\left(R_{2}\right)=0 ; 2\right) d P_{4} / d r=1$ at $\left.r=R_{1} ; 3\right) \int_{R_{1}}^{R_{2}} r P_{4}(r) d r=0$. We take

$$
P_{4}(r)=\frac{1}{R_{1}^{2}\left(R_{1}-R_{2}\right)}\left(r^{2}-\left(R_{1}+R_{2}\right) r+R_{1} R_{2}\right)\left(r^{2}+C_{1} r+C_{2}\right)
$$

with constants

$$
C_{1}=-\frac{\left(R_{1}+R_{2}\right)\left(2 R_{1}^{2}+2 R_{2}^{2}+R_{1} R_{2}\right)}{\left(R_{2}-R_{1}\right)\left(3 R_{2}+2 R_{1}\right)}, \quad C_{2}=-R_{1} C_{1} .
$$

We explain the construction of the polynomial $P_{4}(r)$ briefly. The polynomial $P_{4}=g\left(R_{1}, R_{2}\right) \times$ $\times(r-a)(r-b)\left(r^{2}+C_{1} r+C_{2}\right)$ follows from the condition 1). Selecting $g\left(R_{1}, R_{2}\right)=\left[R_{1}^{2}\left(R_{1}-R_{2}\right)\right]^{-1}$ from condition 2) we obtain the equality $R_{1} C_{1}+C_{2}=0$. The another equation for $C_{1}$ and $C_{2}$ follows from equality 3 ):

$$
\left(3 R_{1}^{2}+3 R_{2}^{2}+4 R_{1} R_{2}\right) C_{1}+5\left(R_{1}+R_{2}\right) C_{2}=-\left(R_{1}+R_{2}\right)\left(2 R_{1}^{2}+2 R_{1}^{2}+R_{2} R_{1}\right),
$$

whence the formulas (3.3) can be obtained.

When change (3.1) is used, boundary conditions (1.3), (1.5) remain the same (of course, we should use the function $\bar{v}_{2}$ instead of function $\left.v_{2}\right)$. The equation for the $\bar{v}_{2}(r, t)$ is inhomogeneous and has the form

$$
\begin{aligned}
\bar{v}_{2 t}=\nu_{2}\left(\bar{v}_{2 r r r}+\frac{1}{r} \bar{v}_{2 r r}\right) & -\frac{2 \nu_{2} \circledast}{\mu_{2}} a_{1}\left(R_{1}, t\right)\left(P_{4 r r}+\frac{1}{r} P_{4 r}\right)+\frac{2 \circledast}{\mu_{2}} a_{1 t}\left(R_{1}, t\right) P_{4}(r)+f_{2}(t) \equiv \\
& \equiv \nu_{2}\left(\bar{v}_{2 r r r}+\frac{1}{r} \bar{v}_{2 r r}\right)+f_{2}(t)+Q_{2}(r, t) .
\end{aligned}
$$

Taking into account the second condition in (1.6) we find the initial data for the function $\bar{v}_{2}$ :

$$
\bar{v}_{2}(r, 0)=\frac{2 æ}{\mu_{2}} a_{1}^{0}\left(R_{1}\right) P_{4}(r) \equiv \bar{v}_{2}^{0}(r) .
$$

We multiply equation (1.1) by $r \rho_{1} v_{1}$, equation (3.4) by $r \rho_{2} v_{2}$, integrate them through domain and sum the results. We obtain

$$
\begin{gathered}
\frac{d E}{d t}+\mu_{1} \int_{0}^{R_{1}} r v_{1 r}^{2} d r+\mu_{2} \int_{R_{1}}^{R_{2}} r \bar{v}_{2 r}^{2} d r=\rho_{2} \int_{R_{1}}^{R_{2}} r \bar{v}_{2} Q_{2} d r-\frac{2 æ a_{1}\left(R_{1}, t\right)}{R_{1}} \int_{0}^{R_{1}} r v_{1} d r \\
E(t)=\frac{\rho_{1}}{2} \int_{0}^{R_{1}} r v_{1}^{2} d r+\frac{\rho_{2}}{2} \int_{R_{1}}^{R_{2}} r \bar{v}_{2}^{2} d r
\end{gathered}
$$

The first condition in (1.3)-(1.5), and equality (1.7) are used to derive identity (3.6). 
The left-hand side of (3.6) is greater than or equal to

$$
\frac{d E}{d t}+\frac{1}{\mathrm{M}}\left(\int_{0}^{R_{1}} r v_{1}^{2} d r+\int_{R_{1}}^{R_{2}} r \bar{v}_{2 r}^{2} d r\right)
$$

with the constant $\mathrm{M}=R_{1}^{2}\left(\mu_{1} x_{0}^{2}\right)^{-1}$, where $x_{0}$ is the smallest positive root of the described above transcendental equation $(2.5)$ with $\gamma_{2}=\sqrt{\mu_{1} / \mu_{2}}=\sqrt{\mu}$.

The right-hand side of (3.6) does not exceed

$$
\left[\sqrt{2 \rho_{2}}\left(\int_{R_{1}}^{R_{2}} r Q_{2}^{2} d r\right)^{1 / 2}+\frac{2 æ}{\sqrt{\rho_{1}}}\left|a_{1}\left(R_{1}, t\right)\right|\right] \sqrt{E(t)}
$$

From (3.7) and (3.8) we obtain the inequality

$$
\frac{d E}{d t}+2 \delta E \leqslant 2 \sqrt{E}\left[\sqrt{\frac{\rho_{2}}{2}}\left(\int_{R_{1}}^{R_{2}} r Q_{2}^{2} d r\right)^{1 / 2}+\frac{\Re}{\sqrt{\rho_{1}}}\left|a_{1}\left(R_{1}, t\right)\right|\right] \equiv 2 \sqrt{E} H_{1}(t)
$$

with the constant $\delta=\mathrm{M}^{-1} \min \left(\rho_{1}^{-1}, \rho_{2}^{-1}\right)$. From (3.9) we find the estimate of function $E(t)$ :

$$
E(t) \leqslant\left[\sqrt{E(0)}+\int_{0}^{t} H_{1}(\tau) e^{\delta \tau} d \tau\right]^{2} e^{-2 \delta t}
$$

where, according to the first equality in (1.6) and (3.5),

$$
E(0)=\frac{\rho_{2}}{2} \int_{R_{1}}^{R_{2}} r \bar{v}_{2}^{0}(r) d r=\frac{2 æ^{2} \rho_{2}}{\mu_{2}^{2}}\left(a_{1}^{0}\left(R_{1}\right)\right)^{2} \int_{R_{1}}^{R_{2}} r P_{4}^{2}(r) d r .
$$

Thus, the estimates of the quantities $v_{1}$ and $\bar{v}_{2}$ in $\mathrm{L}_{2}$-norms $\forall t \in[0, T]$ follow from (3.10) where $\alpha(t)$ and $\alpha^{\prime}(t)$ are given.

To evaluate the derivatives of $v_{1 r}, \bar{v}_{2 r}$ in $\mathrm{L}_{2}$-norm we multiply (1.1) by $r \rho_{1} v_{1 t}$ and equation (3.4) by $r \rho_{2} \bar{v}_{2 t}$, integrate by their domains and sum up the results. We obtain identity

$$
\begin{aligned}
\rho_{1} \int_{0}^{R_{1}} v_{1 t}^{2} d r+\rho_{2} \int_{R_{1}}^{R_{2}} \bar{v}_{2 t}^{2} d r+\frac{1}{2} \frac{\partial}{\partial t} & {\left[\mu_{1} \int_{0}^{R_{1}} r v_{2 r}^{2} d r+\mu_{2} \int_{R_{1}}^{R_{2}} r \bar{v}_{1 r}^{2} d r\right]=} \\
& =\rho_{2} \int_{R_{1}}^{R_{2}} r \bar{v}_{2 t} Q_{2} d r-\frac{2 \Re}{R_{1}} a_{1}\left(R_{1}, t\right) \int_{0}^{R_{1}} r v_{1 t} d r
\end{aligned}
$$

where $Q_{2}(r, t)$ is defined in (3.4). We estimate the right-hand side of (3.12)

$$
\frac{\rho_{2}}{2} \int_{R_{1}}^{R_{2}} r \bar{v}_{2 t}^{2} d r+\frac{\rho_{2}}{2} \int_{R_{1}}^{R_{2}} r Q_{2}^{2} d r+\frac{\Re^{2}}{2 \rho_{1}} a_{1}^{2}\left(R_{1}, t\right)+\rho_{1} \int_{0}^{R_{1}} r \bar{v}_{1 t}^{2} d r .
$$

Therefore, from (3.12) we obtain the required inequality

$$
\begin{aligned}
\mu_{1} \int_{0}^{R_{1}} r v_{1 r}^{2} d r+\mu_{2} \int_{R_{1}}^{R_{2}} r \bar{v}_{2 r}^{2} d r & \leqslant \mu_{2} \int_{R_{1}}^{R_{2}} r\left(\bar{v}_{2 r}^{0}\right)^{2} d r+ \\
& +\frac{\rho_{2}}{2} \int_{0}^{t} \int_{R_{1}}^{R_{2}} r Q_{2}^{2}(r, t) d r d t+\frac{\Re^{2}}{\rho_{1}} \int_{0}^{t} a_{1}^{2}\left(R_{1}, t\right) d t \equiv H_{2}(t)
\end{aligned}
$$


whence the limitation of the derivatives $v_{1 r}, \bar{v}_{2 r}$ follows in $\mathrm{L}_{2}$-norms $\forall t \in[0, T]$.

Also, as in 2., we have

$$
\begin{gathered}
\bar{v}_{2}^{2}(r, t)=-2 \int_{r}^{R_{2}} \bar{v}_{2} \bar{v}_{2 r}^{2} d r \leqslant \frac{2}{R_{1}}\left(\int_{R_{1}}^{R_{2}} r \bar{v}_{2}^{2} d r\right)^{1 / 2}\left(\int_{R_{1}}^{R_{2}} r \bar{v}_{2 r}^{2} d r\right)^{1 / 2} \leqslant \\
\leqslant \frac{2}{R_{1}}\left(\frac{H_{2}(t)}{\mu_{2}}\right)^{1 / 2}\left(\frac{2}{\rho_{2}} E(t)\right)^{1 / 2} .
\end{gathered}
$$

Thus, $\forall t \in[0, T], r \in\left[R_{1}, R_{2}\right]$ the estimate is valid

$$
\left|\bar{v}_{2}(r, t)\right| \leqslant \sqrt{\frac{2}{R_{1}}}\left(\frac{2}{\rho_{2} \mu_{2}} H_{2}(t) E(t)\right)^{1 / 4},
$$

where $H_{2}(t)$ is the right-hand side of inequality (3.13) and the function $E(t)$ is estimated by expression (3.10). Taking into account the replacement of (3.1), we obtain the estimate

$$
\left|v_{2}(r, t)\right| \leqslant \frac{2 æ}{\mu_{2}}\left|a_{1}\left(R_{1}, t\right)\right| \max _{r \in\left[R_{1}, R_{2}\right]}\left|P_{4}(r)\right|+\sqrt{\frac{2}{R_{1}}}\left(\frac{2}{\rho_{2} \mu_{2}} H_{2}(t) E(t)\right)^{1 / 4} .
$$

Similarly, differentiating problem for $v_{1}(r, t), v_{2}(r, t)$ by $t$, we get a priori estimate of form (3.15):

$$
\left|v_{2 t}(r, t)\right| \leqslant \frac{2 æ}{\mu_{2}}\left|a_{1 t}\left(R_{1}, t\right)\right| \max _{r \in\left[R_{1}, R_{2}\right]}\left|P_{4}(r)\right|+\sqrt{\frac{2}{R_{1}}}\left(\frac{2}{\rho_{2} \mu_{2}} H_{3}(t) E_{1}(t)\right)^{1 / 4},
$$

where $E_{1}(t)$ is different from $E(t)$ that $v_{1}, v_{2}$ should be replaced by $v_{1 t}, v_{2 t} ; H_{3}(t)$ is different from $H_{2}(t)$ that $a_{1}\left(R_{1}, t\right), a_{1 t}\left(R_{1}, t\right)$ should be replaced by $a_{1 t}\left(R_{1}, t\right), a_{1 t t}\left(R_{1}, t\right)$, respectively. These estimates, in view of the first equality in (1.6) and the inequalities $(2.20),(2.21)$, are already known.

To estimate $\left|v_{1}(r, t)\right|$, we proceed as follows. We consider the problem

$$
\begin{gathered}
v_{1 t}=\nu_{1}\left(v_{1 r r}+\frac{1}{r} v_{1 r}\right)+f_{1}(t), \quad 0<r<R_{1}, \\
v_{1}\left(R_{1}, t\right)=v_{2}\left(R_{1}, t\right), \quad\left|v_{1}(0, t)\right|<\infty, \quad v_{1}(r, 0)=0,
\end{gathered}
$$

assuming $v_{2}\left(R_{1}, t\right)$ to be known, satisfying estimate $(3.15)$. The solution of this problem is given by formula [11]

$$
\begin{aligned}
& v_{1}(r, t)=\frac{2 \nu_{1}}{R_{1}} \sum_{n=1}^{\infty} \frac{\xi_{n} J_{0}\left(\xi_{n} r / R_{1}\right)}{J_{1}\left(\xi_{n}\right)} \int_{0}^{t} v_{2}\left(R_{1}, \tau\right) \exp \left(-\frac{\nu_{1} \xi_{n}^{2}(t-\tau)}{R_{1}^{2}}\right) d \tau+ \\
& \quad+\frac{2}{R_{1}} \sum_{n=1}^{\infty} \frac{J_{0}\left(\xi_{n} r / R_{1}\right)}{\xi_{n} J_{1}\left(\xi_{n}\right)} \int_{0}^{t} f_{1}(\tau) \exp \left(-\frac{\nu_{1} \xi_{n}^{2}(t-\tau)}{R_{1}^{2}}\right) d \tau
\end{aligned}
$$

$\xi_{n}$ are the roots of the Bessel function $J_{0}\left(\xi_{n}\right)=0$.

Note that $f_{1}(t)$ is unknown; we find its relationship with $v_{1}$. Multiplying the equation (3.16) by $r$ and integrating from 0 to $R_{1}$, we find

$$
f_{1}(t)=-2 \nu_{1} v_{1 r}\left(R_{1}, t\right)+\frac{2}{R_{1}^{2}} \frac{\partial}{\partial t} \int_{0}^{R_{1}} r v_{1} d r=-2 \nu_{1} v_{1 r}\left(R_{1}, t\right)-\frac{2}{R_{1}^{2}} \int_{R_{1}}^{R_{2}} r v_{2 t} d r
$$


where the second equality (1.3) is used. In equality (3.20) we do not have an estimate for the first summand, the second can be estimated using inequality (3.16). We differentiate equation (3.17) with respect to $r$ and introduce a new function $V(r, t)=v_{1 r}(r, t)$. We obtain the equation for it following:

$$
V_{t}=\nu_{1}\left(V_{r r}+\frac{1}{r} V_{r}-\frac{1}{r^{2}} V\right),
$$

where $|V(0, t)|<\infty$. The second boundary condition for $V$ is found from the consideration of the integral

$$
\begin{aligned}
\int_{0}^{R_{1}} r^{2} V d r & =\int_{0}^{R_{1}} r^{2} v_{1 r} d r=R_{1}^{2} v_{1}\left(R_{1}, t\right)-2 \int_{0}^{R_{1}} r v_{1} d r= \\
& =R_{1}^{2} v_{2}\left(R_{1}, t\right)+2 \int_{R_{1}}^{R_{2}} r v_{2} d r \equiv g(t)
\end{aligned}
$$

with known a priori estimate function $g(t), g(0)=0$ (we use the second equation applied (1.3).

We change the function $V$ :

$$
V(r, t)=\bar{V}(r, t)+\left(r^{4}-\frac{6}{7} R_{1} r^{3}\right) g(t) .
$$

The problem for $\bar{V}(r, t)$ takes the form (it is nonclassical)

$$
\begin{gathered}
\bar{V}_{t}=\nu_{1}\left(\bar{V}_{r r}+\frac{1}{r} \bar{V}_{r}-\frac{1}{r^{2}} \bar{V}\right)+\nu_{1}\left(15 r^{2}-\frac{48}{7} R_{1} r\right) g(t)+\left(\frac{6}{7} R_{1} r^{3}-r^{4}\right) g_{t}(t) \\
\bar{V}(r, 0)=0, \quad \int_{0}^{R_{1}} r^{2} \bar{V} d r=0, \quad|\bar{V}(0, t)|<\infty
\end{gathered}
$$

Lemma 3.1. Solution (3.24), (3.25) has form

$$
\bar{V}(r, t)=\sum_{n=1}^{\infty} \bar{V}_{n}(t) J_{1}\left(\frac{\zeta_{n} r}{R_{1}}\right)
$$

where $\zeta_{n}$ are the positive roots of equation $J_{2}(\zeta)=0$ and we obtain

$$
\bar{V}_{n}(t)=g(t) h_{n}^{2}+\left(\nu_{1} h_{n}^{1}-\frac{\nu_{1} \zeta_{n}^{2}}{R_{1}^{2}} h_{n}^{2}\right) \int_{0}^{t} g(\tau) \exp \left[-\frac{\nu_{1} \zeta_{n}^{2}}{R_{1}^{2}}(t-\tau)\right] d \tau
$$

where $h_{n}^{1}$ and $h_{n}^{2}$ are defined by formulas

$$
\begin{aligned}
h_{n}^{1} & =\frac{2}{R_{1}^{2} J_{1}^{2}\left(\zeta_{n}\right)} \int_{0}^{R_{1}}\left(15 r^{3}-\frac{48}{7} R_{1} r^{2}\right) J_{1}\left(\frac{\zeta_{n}}{R_{1}} r\right) d r \\
h_{n}^{2} & =\frac{2}{R_{1}^{2} J_{1}^{2}\left(\zeta_{n}\right)} \int_{0}^{R_{1}}\left(\frac{6}{7} R_{1} r^{4}-r^{5}\right) J_{1}\left(\frac{\zeta_{n}}{R_{1}} r\right) d r .
\end{aligned}
$$

Proof. Since the formula is valid [9]

$$
\int_{0}^{z} \tau^{k} J_{k-1}(\tau) d \tau=z^{k} J_{k}(z)
$$

here $k \geqslant 1$ is integer. Then the solution of problem (3.24), (3.25) should be searched for as a Fourier series (we have $k=2$ ) $(3.26)$, since [12] $\zeta J_{1}^{\prime}(\zeta)-J_{1}(\zeta)=-\zeta J_{2}(\zeta)$, the roots of the 
equation $J_{2}(\zeta)=0$ are the roots of function $\zeta J_{1}^{\prime}(\zeta)-J_{1}(\zeta)$. The presentation of (3.26) takes place [12].

Substitution of (3.26) in (3.24), (3.25) leads to the Cauchy problem

$$
\bar{V}_{n t}+\frac{\nu_{1} \zeta_{n}^{2}}{R_{1}^{2}} \bar{V}_{n}=\nu_{1} g(t) h_{n}^{1}+g_{t}(t) h_{n}^{2}, \quad \bar{V}_{n}(0)=0,
$$

where $h_{n}^{1}, h_{n}^{2}$ are coefficients of Fourier series of functions $15 r^{2}-48 R_{1} r / 7,6 R_{1} r^{3} / 7-r^{4}$ respectively, defined by formulas (3.28). From (3.29) we find that the solution of problem (3.24), (3.25) has form (3.27).

Taking into account replacement (3.23), we obtain an expression for the function $v_{1 r}\left(R_{1}, t\right)$ :

$$
\begin{aligned}
v_{1 r}\left(R_{1}, t\right)=\bar{V}\left(R_{1}, t\right) & +\frac{1}{7} R_{1}^{4} g(t)=\left(\frac{1}{7} R_{1}^{4}+\sum_{n=1}^{\infty} h_{n}^{2} J_{1}\left(\zeta_{n}\right)\right) g(t)+ \\
+ & \sum_{n=1}^{\infty}\left(\nu_{1} h_{n}^{1}-\frac{\nu_{1} \zeta_{n}^{2}}{R_{1}^{2}} h_{n}^{2}\right) J_{1}\left(\zeta_{n}\right) \int_{0}^{t} g(\tau) \exp \left[-\frac{\nu_{1} \zeta_{n}^{2}}{R_{1}^{2}}(t-\tau)\right] d \tau
\end{aligned}
$$

The series in (3.30) and series (3.26) converge uniformly. Really we have

$$
\begin{gathered}
h_{n}^{1}=\frac{2}{R_{1} \zeta_{n} J_{1}^{2}\left(\zeta_{n}\right)} \int_{0}^{R_{1}}\left(15 r-\frac{4}{7} R_{1}\right) \frac{d}{d r}\left(r^{2} J_{2}\left(\frac{\zeta_{n}}{R_{1}} r\right)\right) d r= \\
=-\frac{30}{R_{1} \zeta_{n} J_{1}^{2}\left(\zeta_{n}\right)} \int_{0}^{R_{1}} r^{2} J_{2}\left(\frac{\zeta_{n}}{R_{1}} r\right) d r \\
h_{n}^{2}=\frac{6}{R_{1} \zeta_{n} J_{1}^{2}\left(\zeta_{n}\right)} \int_{0}^{R_{1}}\left(r^{4}-\frac{4}{7} R_{1} r^{3}\right) J_{2}\left(\frac{\zeta_{n}}{R_{1}} r\right) d r .
\end{gathered}
$$

Since the equality $J_{k-1}(z)+J_{k+1}(z)=2 k z^{-1} J_{k}(z)$ is valid [9], so we obtain $J_{3}\left(\zeta_{n}\right)=-J_{1}\left(\zeta_{n}\right)$ (we recall, that $J_{2}\left(\zeta_{n}\right)=0$ ) and so the expression for the function $h_{n}^{1}$ and $h_{n}^{2}$ have following the form

$$
h_{n}^{1}=\frac{\beta_{n}^{1}}{\zeta_{n}}, \quad h_{n}^{2}=\frac{\beta_{n}^{2}}{\zeta_{n}}
$$

where $\beta_{n}^{1}, \beta_{n}^{2}$ are coefficients of Fourier series of functions $-15 R_{1} r$ and $3 R_{1}\left(r^{3}-4 R_{1} r^{2} / 7\right)$ when they are decomposed by function $J_{2}\left(R_{1}^{-1} \zeta_{n} r\right)$. The series $\sum_{n=1}^{\infty}\left(\beta_{n}^{j}\right)^{2}$ converge, and then, by virtue of inequality $\left|h_{n}^{j}\right| \leqslant 2^{-1}\left[\left(\beta_{n}^{j}\right)^{2}+1 / \zeta_{n}^{2}\right]$, the series $\sum_{n=1}^{\infty}\left|h_{n}^{j}\right|$ also converge. The series $\sum_{n=1}^{\infty} \zeta_{n}^{-2}$ converge, since $\zeta_{n} \sim n \pi$ at $n \gg 1$. Moreover we obtain [11] $\sum_{n=1}^{\infty} 1 / n^{2} \pi^{2}=1 / 12$.

As for the second term in (3.30) its is less or equal then to

$$
R_{1}^{2} \sum_{n=1}^{\infty}\left(\frac{\left|h_{n}^{1}\right|}{\zeta_{n}^{2}}+\frac{\left|h_{n}^{2}\right|}{R_{1}^{2}}\right) \max _{t \in[0, T]}|g(t)|
$$

with obviously convergent series.

Remark 3. In the preceding arguments we used the well-known inequality $\left|J_{k}(z)\right| \leqslant 1$, where the constant $k>0$ is integer [9]. 
Remark 4. Following the monographs [10,12], we can show that the function $\bar{V}(r, t)$ is the sum of series (3.26) and it has derivatives of all orders on $r$ and $t$ at $t \geqslant \varepsilon>0$. In particular, the solution of problem (3.24), (3.25) is classical.

From (3.16), (3.20) and (3.30) we find estimate $f_{1}(t)$ at $t \in[0, T]$ :

$$
\begin{aligned}
\left|f_{1}(t)\right| \leqslant 2 \nu_{1}\left[\left(\frac{1}{7} R_{1}^{4}+\sum_{n=1}^{\infty}\left|h_{n}^{2}\right|\right)+2 R_{1}^{2} \sum_{n=1}^{\infty}\left(\frac{\left|h_{n}^{1}\right|}{\zeta_{n}^{2}}+\frac{\left|h_{n}^{2}\right|}{R_{1}^{2}}\right)\right] \max _{t \in[0, T]}|g(t)|+ \\
+\frac{R_{2}^{2}-R_{1}^{2}}{R_{1}^{2}}\left[\frac{2 æ}{\mu_{2}} \max _{t \in[0, T]}\left|a_{1 t}\left(R_{1}, t\right)\right| \max _{r \in\left[R_{1}, R_{2}\right]}\left|P_{4}(r)\right|+\right. \\
\left.+\sqrt{\frac{2}{R_{1}}} \max _{t \in[0, T]}\left(\frac{2}{\rho_{2} \mu_{2}} H_{3}(t) E_{1}(t)\right)^{1 / 4}\right]
\end{aligned}
$$

The estimate $f_{2}(t)$ follows from (1.7)

$$
\left|f_{2}(t)\right| \leqslant \rho\left|f_{1}(t)\right|+\frac{2 æ}{\rho_{2} R_{1}} \max _{t \in[0, T]}\left|a_{1}\left(\rho_{2} R_{1}, t\right)\right|,
$$

where $\rho=\rho_{1} / \rho_{2}$ and $f_{1}(t)$ satisfies inequality (3.33).

Thus, the functions $f_{j}(t)$ are bounded and continuous at $t \in[0, T]$.

The limitation of the function $v_{1}(r, t)$ at $r \in\left[0, R_{1}\right], t \in[0, T]$ follows from it representation in the form (3.19) (the equality (2.28) is used)

$$
\left|v_{1}(r, t)\right| \leqslant R_{1} \max _{t \in[0, T]}\left|v_{2}\left(R_{1}, t\right)\right|+\frac{2 R_{1}}{\nu_{1}} \max _{t \in[0, T]}\left|f_{1}(t)\right| \sum_{n=1}^{\infty} \frac{1}{\xi_{n}^{3}\left|J_{1}\left(\xi_{n}\right)\right|},
$$

where the functions modules $\left|v_{2}\left(R_{1}, t\right)\right|,\left|f_{1}(t)\right|$ satisfies estimates (3.15) and (3.33). The series in (3.35) converge, since $\xi_{n} \sim n \pi,\left|J_{1}\left(\xi_{n}\right)\right| \sim 1 / \sqrt{n}$ at $n \gg 1$.

Thus, we have

Theorem 3.1. The solutions of the initial-boundary value problem (1.1)-(1.7), (1.15) $v_{j}(r, t)$ and the function $f_{j}(t)$ are limitation for all $r \in\left[0, R_{1}\right](j=1)$ and $r \in\left[R_{1}, R_{2}\right](j=2)$, and $t \in[0, T]$. Estimates (3.15), (3.35) are valid for function $v_{j}(r, t)$ and estimates (3.33), (3.34) take place for function $f_{j}(t)$.

\section{Conclusion}

One partially invariant solution of the equation describing the axisymmetric motion of a viscous heat-conducting liquid is studied. As a result, the unimprovable uniform priori estimates for the solutions of the conjugate problem posed are obtained for small Marangoni number.

\section{References}

[1] V.K.Andreev, V.E.Zahvataev, E.A.Ryabitskii, Thermocapillary Instability, Novosibirsk, Nauka, (2000) (in Russian).

[2] A.Nepomnyashii, I.Simanovskii, J.-C.Legros, Interfacial Convection in Multilayer System, New-York, Springer, 2006. 
[3] R.Narayanan, D.Schwabe, Interfacial Fluid Gynamics and Transport Processes, Berlin, Heidelberg, New-York, Springer-Verlag, 2009.

[4] R.Kh.Zeytovnian, Convection in Fluids, Dordrecht, Heidelberg, London, New-York, Springer, 2009.

[5] K.Hiemenz, Die grenzschicht an einem in den gleichformigen Flussigkeitsstrom eingetauchten graden Kreiszylinder, Dinglers Polytech. J., 326(1911), 321-440.

[6] J.F.Brady, A.Acrivos, Steady flow in a channel or tube with an accelerating surface velocity, J. Fluid Mech., 112(1981), 127-150.

[7] V.K.Andreev, N.L.Sobachkina, The motion of a binary mixture in planar and cylindrical regions, Krasnoyarsk, Siberian Federal University, 2012.

[8] V.K.Andreev On the Friedrichs inequality for compound domains, J. Sib. Fed. Univ. Math. and phys., 2(2009), 146-157 (in Russian).

[9] G.Bateman, A.Erdein, Higher transcendental functions. Bessel functions, parabolic cylinder functions, orthogonal polynomials, Moscow, Nauka, 1974 (in Russian).

[10] S.G.Mikhlin, Linear partial differential equations, Moscow, High school, 1977 (in Russian).

[11] A.P.Prudnikov, Y.A.Bychkov, O.I.Marichev, Integrals and series. Special functions, Moscow, Nauka, 1983 (in Russian).

[12] V.S.Vladimirov, Equations of mathematical physics, Moscow, Nauka, 1976 (in Russian).

\title{
Априорные оценки сопряжённой задачи, описывающей осесимметричное термокапиллярное движение при малых числах Марангони
}

\author{
Виктор К. Андреев \\ Евгений П. Магденко \\ Институт вычислительного моделирования СО РАН \\ Академгородок, 50/44, Красноярск, 660036 \\ Институт математики и фундаментальной информатики \\ Сибирский федеральный университет \\ Свободный, 79, Красноярск, 660041 \\ Россия
}

\begin{abstract}
Данная работа посвящена исследованию решения уравнения, описывающего осесимметричное движение вязкой теплопроводной жидкости. Оно интерпретируется как двухслойное движение вязких теплопроводных жидкостей в цилиндре с твёрдой стенкой и общей подвижной недеформируемой поверхностью раздела. С математической точки зрения, возникающая началънокраевая задача является нелинейной и обратной. При некоторых (часто выполняющихся в практических приложения) предположениях задача заменяется линейной. Для неё получены априорные оценки.
\end{abstract}

Ключевые слова: априорные оценки, сопряжённая обратная задача, поверхность раздела, число Марангони. 